\title{
ADUBAÇÃO COM NPK EM COQUEIRO-ANÃO-VERDE (Cocos nucifera L.) RENDIMENTO E QUALIDADE DE FRUTOS ${ }^{1}$
}

\author{
LUIZ ANTONIO JUNQUEIRA TEIXEIRA², ONDINO CLEANTE BATAGLIA ${ }^{3}$, SALATIÉR BUZETTI ${ }^{4}$, \\ ENES FURLANI JUNIOR ${ }^{4}$, JACIRA DOS SANTOS ISEPON ${ }^{4}$
}

\begin{abstract}
RESUMO- A produção de coco para extração de água no Estado de São Paulo está em expansão. Entretanto, faltam informações básicas sobre o manejo desta cultura. Desse modo, instalou-se um experimento de campo em Pereira Barreto (20 $\left.47^{\prime} \mathrm{S} ; 5^{\circ} 01^{\prime} \mathrm{W}\right)$, visando ao estudo da resposta do coqueiro à adubação com NPK de set/2000 a fev/2004. Empregou-se o esquema fatorial fracionado $1 / 2\left(4^{3}\right)$, sendo os tratamentos formados pelas combinações de doses anuais de N $\left(0 ; 120 ; 240\right.$ e $\left.360 \mathrm{~kg} \mathrm{ha}^{-1} \mathrm{de} \mathrm{N}\right), \mathrm{P}\left(0 ; 100 ; 200\right.$ e $\left.300 \mathrm{~kg} \mathrm{ha}^{-1} \mathrm{de}_{2} \mathrm{O}_{5}\right)$ e K $\left(0 ; 120 ; 240\right.$ e $360 \mathrm{~kg} \mathrm{ha}{ }^{-1}$ de $\left.\mathrm{K}_{2} \mathrm{O}\right)$. Como fontes de $\mathrm{N}, \mathrm{Pe} \mathrm{K}$, respectivamente, aplicaram-se $\mathrm{NH}_{4} \mathrm{NO}_{3}$, superfosfato triplo e $\mathrm{KCl}$, fracionadamente, durante a época das chuvas. A aplicação de $\mathrm{N}$ causou diminuição no volume de água e massa média dos frutos e na quantidade de frutos por cacho; inversamente, a adubação potássica teve efeito positivo nestas características. Os teores de $\mathrm{Pe} \mathrm{K}$ da água de coco aumentaram com a aplicação de $\mathrm{Pe} \mathrm{K}$, entretanto não houve efeito dos tratamentos nos resultados da avaliação sensorial. A aplicação de P não teve efeito significativo na produção de frutos.
\end{abstract}

Termos para indexação: nitrogênio, fósforo, potássio, coco

\section{NPK FERTILIZATION ON DWARF GREEN COCONUT (Cocos nucifera L.) - YIELD AND FRUIT QUALITY}

ABSTRACT- Coconut production aiming water extraction is increasing in São Paulo State, Brazil. Coconut is a new crop in this region, so there is a lack of information about its management. A field experiment was conducted in Pereira Barreto, SP, Brazil (20 $\left.47^{\circ} \mathrm{S} ; 51^{\circ} 01^{\prime} \mathrm{W}\right)$ to study the effects of NPK rates on yield and fruit quality, from September,2000 to February, 2004. The experimental design was an incomplete NPK factorial 1/2(43). The treatments were annual rates of $\mathrm{N}\left(0,120,240\right.$, and $360 \mathrm{~kg} \mathrm{ha}^{-1}$ of $\left.\mathrm{N}\right), \mathrm{P}\left(0,100,200\right.$, and $300 \mathrm{~kg} \mathrm{ha}^{-1}$ of $\left.\mathrm{P}_{2} \mathrm{O}_{5}\right)$ and $\mathrm{K}\left(0,120,240\right.$, and $360 \mathrm{~kg}$ ha ${ }^{-1}$ of $\left.\mathrm{K}_{2} \mathrm{O}\right)$, applied as $\mathrm{NH}_{4} \mathrm{NO}_{3}$, triple superphosphate and $\mathrm{KCl}$, respectively. The rates of $\mathrm{N}, \mathrm{Pe} \mathrm{K}$ were split during rainy season. The effect of $\mathrm{N}$ rates was negative on water volume production on fruits, weight and number of fruits per bunch, and in a different way, $\mathrm{K}$ application had a positive effect on them. $\mathrm{P}$ and $\mathrm{K}$ contents in the coconut water increased with $\mathrm{P}$ and $\mathrm{K}$ fertilization, otherwise the treatments had no effect on sensory evaluation results. $P$ application had no effect on fruit production.

Index terms: nitrogen, phosphorus, potassium, coconut.

\section{INTRODUÇÃO}

O cultivo de coqueiro (Cocos nucifera L.) no Estado de São Paulo apresentou significativa expansão nos últimos anos visando a atender à demanda por coco verde para extração de água. Entretanto, tal crescimento tem posto em evidência diversas limitações de ordem agronômica, destacando-se o plantio de materiais com baixa qualidade genética e fitossanitária e a inadequação de parte das técnicas de cultivo às condições regionais. $\mathrm{O}$ pacote tecnológico disponível para a cultura foi desenvolvido baseado nas experiências de plantios do Nordeste brasileiro. Além das diferenças edafoclimáticas, grande parte dos coqueirais da região NE visa à produção de coco-seco, enquanto em São Paulo cultiva-se principalmente coqueiro-anão para extração de água.

Sobral (1998) creditou o baixo rendimento dos coqueiros em algumas regiões do Nordeste à fertilidade do solo e considerou que a adubação seria a prática de maior impacto sobre a produtividade da cultura. O crescimento contínuo do coqueiro implica, segundo Sobral (2003), a remoção de grandes quantidades de nutrientes, os quais necessitam ser repostos por meio da aplicação de fertilizantes. No caso dos 'Anões', esta remoção seria ainda maior porque sua produtividade é superior à dos genótipos cultivados tradicionalmente. Numa revisão sobre manejo de adubação para coqueiro, Magat (2005) apresentou dados de absorção de $\mathrm{N}, \mathrm{P}_{2} \mathrm{O}_{5}$ e $\mathrm{K}_{2} \mathrm{O}$ que chegam, respectivamente, a $174 ; 46$ e $299 \mathrm{~kg} \mathrm{ha}^{-1}$; destes totais, cerca de $62 \%$ do N, $84 \%$ do P e $78 \%$ do K são removidos pelos frutos.

O objetivo deste trabalho foi estudar os efeitos da adubação com nitrogênio, fósforo e potássio na produção de coco para extração de água nas condições de cultivo de São Paulo, visando a subsidiar a elaboração de recomendação de adubação para o Estado.

\section{MATERIALEMÉTODOS}

A área experimental foi estabelecida em Pereira Barreto-SP
(2047'S; 51 $01^{\circ} \mathrm{W}$ ), em fevereiro de 1997, com as plantas ('Anão-verde') arranjadas num espaçamento de 7,5 x 7,5 x 7,5 m (205 plantas/ha). O delineamento experimental foi o de blocos ao acaso, no esquema fatorial fracionado $1 / 2\left(4^{3}\right)$, sendo os tratamentos, as combinações de doses anuais de $\mathrm{N}\left(0 ; 120 ; 240\right.$ e $\left.360 \mathrm{~kg} \mathrm{ha}^{-1} \mathrm{de} \mathrm{N}\right), \mathrm{P}(0 ; 100 ; 200$ e 300 $\mathrm{kg} \mathrm{ha}^{-1}$ de $\left.\mathrm{P}_{2} \mathrm{O}_{5}\right)$ e $\mathrm{K}\left(0 ; 120 ; 240\right.$ e $360 \mathrm{~kg} \mathrm{ha}^{-1}$ de $\left.\mathrm{K}_{2} \mathrm{O}\right)$. As fontes de N, P e K foram, respectivamente, $\mathrm{NH}_{4} \mathrm{NO}_{3}(32 \%$ de $\mathrm{N})$, superfosfato triplo ( $41 \%$ de $\mathrm{P}_{2} \mathrm{O}_{5}$ ) e $\mathrm{KCl}\left(58 \%\right.$ de $\left.\mathrm{K}_{2} \mathrm{O}\right)$. Maiores detalhes do experimento são apresentados por Teixeira et al. (2005). Antes do início da aplicação dos tratamentos (set/2001), o solo da área foi amostrado para análise química realizada segundo os métodos descritos por Raij et al. (2001). Os resultados são apresentados na Tabela 1 .

Em agosto de 2002, junho e outubro de 2003, foram coletados seis frutos por tratamento para pesagem e análises químicas, físicas e de aceitabilidade da água (análise sensorial). Estes frutos foram colhidos aproximadamente sete meses após a abertura da espata. A caracterização física e química da água (albúmen líquido) de coco seguiu as normas analíticas do Instituto Adolfo Lutz (Instituto..., 1985), sendo determinados os sólidos solúveis totais (SST), acidez total titulável (ATT), pH e, por cálculo, obteve-se o ratio (SST/ATT). Os teores de $\mathrm{N}$ mineral ( $\mathrm{N}$ nítrico+N amoniacal), $\mathrm{Ca}, \mathrm{Mg}, \mathrm{P}$ e K na água foram quantificados adaptando-se métodos apresentados por Silva (1999) e Malavolta et al. (1997). A avaliação de aceitabilidade foi realizada por uma equipe de 20 julgadores não treinados que atribuíram notas numa escala de 1 a 9 , sendo a maior nota para as amostras consideradas de melhor sabor.

Nos períodos de dezembro de 2002 a abril de 2003 e de agosto a outubro de 2003, foram realizadas colheitas seguindo os mesmos critérios de coleta de frutos para a comercialização das demais áreas da fazenda. Os frutos, em torno de sete meses após a abertura da espata, eram coletados em intervalos de aproximadamente 30 dias, sendo os resultados destas colheitas expressos em número médio de frutos colhidos por mês.

\footnotetext{
${ }^{1}$ (Trabalho 132/2004). Recebido: 07/10/2004. Aceito para publicação: 19/04/2005. Trabalho realizado com o apoio da FAPESP (Projeto 00/09417-1)

${ }^{2}$ Pesquisador do Instituto Agronômico-IAC. Cx Postal 28, 13001-970, Campinas-SP. teixeira@iac.sp.gov.br

${ }^{3}$ Pesquisador do Instituto Agronômico-IAC, bolsista do CNPq, ondino@iac.sp.gov.br

${ }^{4}$ Professor da FEIS/UNESP, Ilha Solteira-SP, sbuzetti@agr.feis.unesp.br; enes@ agr.feis.unesp.br; jacira@ agr.feis.unesp.br
} 
TABELA 1 - Atributos químicos do solo cultivado com coqueiro, amostragem inicial em setembro de 2000 das camadas de solo de 0 a $20 \mathrm{~cm}$ e de 20 a $40 \mathrm{~cm}$.

\begin{tabular}{|c|c|c|c|c|c|c|c|c|}
\hline Amostragem & MO & $\mathrm{pH}\left(\mathrm{CaCl}_{2}\right)$ & $\mathbf{P}_{\text {resina }}$ & $\mathbf{K}^{+}$ & $\mathrm{Ca}^{2+}$ & $\mathrm{Mg}^{2+}$ & $\mathbf{H}+\mathbf{A l}$ & $\mathbf{V}$ \\
\hline & $\mathrm{g} \mathrm{dm} \mathrm{dm}^{-3}$ & & $\mathrm{mg} \mathrm{dm}^{-3}$ & $\begin{array}{r}--- \\
0\end{array}$ & -----1 & $n^{-3}---$ & ------ & $\%$ \\
\hline Linha ${ }^{1)}$ & 12 & 4,2 & 5 & 2,0 & 2 & 2 & 28 & 17 \\
\hline Entrelinhą) & 15 & 4,9 & 4 & $\begin{array}{r}4,2 \\
20\end{array}$ & 6 & 4 & 20 & 41 \\
\hline Linha & 14 & 4,2 & 3 & 1,5 & 3 & 2 & 30 & 18 \\
\hline Entrelinha & 12 & 4,9 & 4 & 2,9 & 6 & 5 & 20 & 40 \\
\hline
\end{tabular}

(1) Área sob a projeção da copa e com aplicação de adubos; ${ }^{(2)}$ área com pouca influência do sistema radicular e sem aplicação de adubos.

Os efeitos dos tratamentos foram avaliados empregando-se o teste F. Para as situações nas quais se detectaram efeitos significativos dos tratamentos de adubação $(p<0,10)$, foram ajustadas equações de regressão relacionando doses de $\mathrm{N}, \mathrm{P}$ ou $\mathrm{K}$ com as variáveis-resposta.

\section{RESULTADOS E DISCUSSÃO}

Os teores de $\mathrm{P}$ e $\mathrm{K}$ da água de coco foram influenciados pela adubação. Nas três épocas, os efeitos da aplicação de $\mathrm{P}$ e K na concentração destes nutrientes na água foram positivos, conforme as regressões apresentadas na Tabela 2. Em média, cada aumento de $100 \mathrm{~kg} \mathrm{ha}^{-1}$ na dose de $\mathrm{P}_{2} \mathrm{O}_{5}$ implicou incremento de $4,0 \mathrm{mg} \mathrm{L}^{-1}$ no teor de $\mathrm{P}$ da água (média dos ajustes lineares). Para potássio, a cada $100 \mathrm{~kg}$ ha ${ }^{1}$ de $\mathrm{K}_{2} \mathrm{O}$, a variação no teor de $\mathrm{K}$ na água foi de 7,0 $\mathrm{mg} \mathrm{L}^{-1}$. Na Figura 1, é apresentada a variação dos teores de $\mathrm{N}$ mineral, $\mathrm{P}$ e K da água para cada dose de adubo ao longo do tempo. Estes resultados sugerem que poderia haver efeitos da adubação no sabor da água, entretanto não se verificou variação significativa da aceitabilidade da água em função dos tratamentos (Tabela 2).

As concentrações de cálcio, nas três amostragens, e de magnésio (exceto na $2^{\mathrm{a}}$ amostragem) na água de coco não foram influenciadas pelos tratamentos de adubação (Tabela 3). A redução do teor de $\mathrm{Mg}$ pode ser creditada à acidificação do solo determinada pela adubação nitrogenada (Teixeira et al., 2005), a qual diminui a disponibilidade deste nutriente.
O pH da água e o teor de sólidos solúveis totais (SST) não foram influenciados pelos tratamentos (Tabelas 3 e 4). A acidez total titulável (ATT), na terceira época de amostragem, diminuiu com a adubação potássica, e o ratio variou de forma inversa (Tabela 4) nesta amostragem. Provavelmente, devido aos efeitos relativamente pequenos da adubação nestes atributos da água, os resultados da análise sensorial também não variaram em função dos tratamentos (Tabela 2). Os valores médios de $\mathrm{pH}$ da água observados foram semelhantes aos apresentados por Lima et al. (2004) para frutos colhidos sete meses após a emissão do cacho; já o teor médio de SST $\left(7,3^{\circ}\right.$ Brix $)$ foi superior ao observado por estes autores (em torno de $5,5^{\circ} \mathrm{Brix}$ ).

Comercialmente, o volume de água de cada fruto é uma característica importante. A quantidade de água diminuiu com o aumento da dose de N, enquanto a aplicação de K teve efeito positivo (Tabela 3 ). Além de diminuir o conteúdo de água, a adubação nitrogenada afetou a massa dos frutos e o número de frutos por cacho (Tabela 5). Houve redução na massa dos frutos e no número de frutos por cacho proporcional às doses de N. De outra forma, a aplicação de potássio aumentou a massa média dos frutos. Sobral \& Leal (1999), diferentemente, observaram que tanto a aplicação de $\mathrm{N}$ como de $\mathrm{K}$ determinaram aumento significativo no número de frutos produzidos.

O número médio de frutos colhidos por mês não foi influenciado pelos tratamentos de adubação (Tabela 5). Provavelmente, esta resposta se deva aos níveis de produtividade alcançados. Em média, colheram-se 7,0 frutos/mês de dezembro a abril e 5,9 frutos/mês de agosto a outubro,
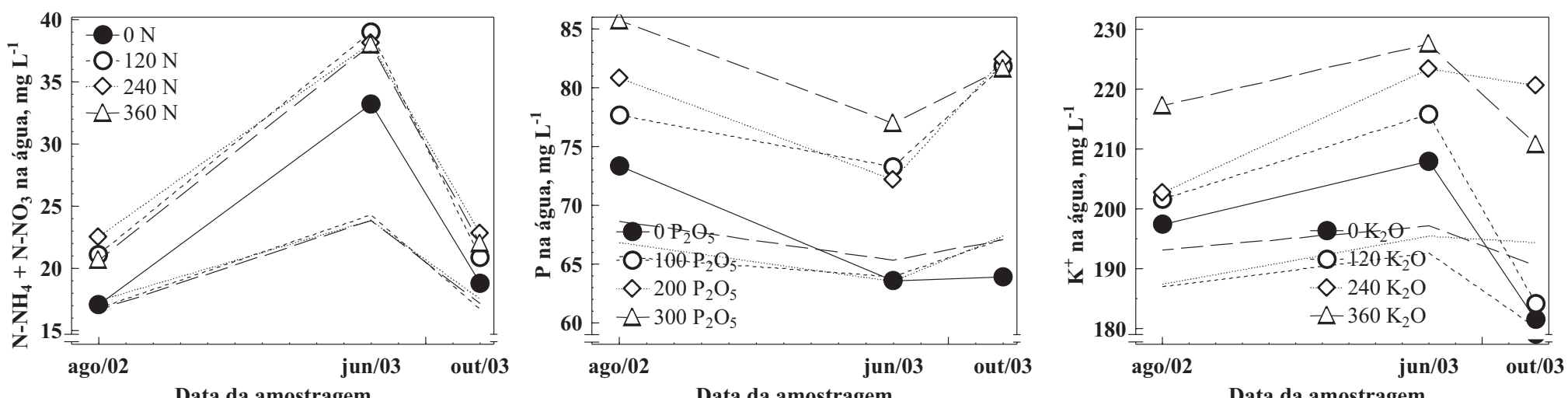

FIGURA 1 - Teores de $\mathrm{N}$ mineral, $\mathrm{P}$ e K da água de coco, considerando dose de adubo ( $\mathrm{kg} \mathrm{ha}^{-1} \mathrm{de} \mathrm{N}, \mathrm{P}_{2} \mathrm{O}_{5}$ e $\left.\mathrm{K}_{2} \mathrm{O}\right)$ e época de amostragem.

TABELA 2 - Efeito da aplicação de $\mathrm{N}, \mathrm{Pe} \mathrm{K}$ (doses em kg ha-1 de N, $\mathrm{P}_{2} \mathrm{O}_{5}$ e $\mathrm{K}_{2} \mathrm{O}$ ) nos teores de $\mathrm{N}$ mineral, $\mathrm{P}$, $\mathrm{K}$ e análise sensorial da água de coco em três épocas de amostragem.

\begin{tabular}{|c|c|c|c|c|c|c|c|c|}
\hline \multicolumn{3}{|c|}{$\mathbf{N}\left(\mathrm{mg} \mathrm{L}^{-1}\right)$} & \multicolumn{2}{|l|}{$\mathbf{P}\left(\mathrm{mg} \mathrm{L}^{-1}\right)$} & \multicolumn{2}{|l|}{$K\left(\mathrm{mg} \mathrm{L}^{-1}\right)$} & \multicolumn{2}{|c|}{ Sensorial (nota) } \\
\hline & Função & $p^{(1)}$ & Função & $p$ & Função & $p$ & Função & $p$ \\
\hline( & $=20,3)-\mathrm{ns}^{(2)}$ & $>0,1$ & $\hat{\mathrm{Y}}=73,38+4,01 \times 10^{-2} \mathrm{P}$ & $<0,0419$ & $\hat{\mathrm{Y}}=195,62+5,06 \times 10^{-2} \mathrm{~K}$ & $<0,0255$ & $(\overline{\mathrm{Y}}=6,6)-\mathrm{ns}$ & $>0,1$ \\
\hline( & $=37,1)-\mathrm{ns}$ & $>0,1$ & $\hat{\mathrm{Y}}=65,63+3,89 \times 10^{-2} \mathrm{P}$ & $<0,0003$ & $\hat{\mathrm{Y}}=208,72+5,53 \times 10^{-2} \mathrm{~K}$ & $<0,0018$ & $(\overline{\mathrm{Y}}=6,3)-\mathrm{ns}$ & $>0,1$ \\
\hline( & $=21,1)-\mathrm{ns}$ & $>0,1$ & $\hat{\mathrm{Y}}=64,71+1,94 \times 10^{1} \mathrm{P}-4,68 \times 10^{4} \mathrm{P}^{2}$ & $<0,0059$ & $\hat{\mathrm{Y}}=180,66+1,03 \times 10^{-1} \mathrm{~K}$ & $<0,0340$ & $(\overline{\mathrm{Y}}=5,9)-\mathrm{ns}$ & $>0,1$ \\
\hline
\end{tabular}

${ }^{(1)}$ Probabilidade de erro tipo I associada ao teste $\mathrm{F}$ (significância do modelo); ${ }^{(2)}$ valor entre parêntesis corresponde à média da variável; ajuste não significativo ( $\left.p>0,1\right)$. 
TABELA 3 - Efeito da aplicação de N, Pe K (doses em kg ha-1 de N, $\mathrm{P}_{2} \mathrm{O}_{5}$ e $\mathrm{K}_{2} \mathrm{O}$ ) nos teores de Ca, $\mathrm{Mg}$, volume e pH da água de coco em três épocas de amostragem.

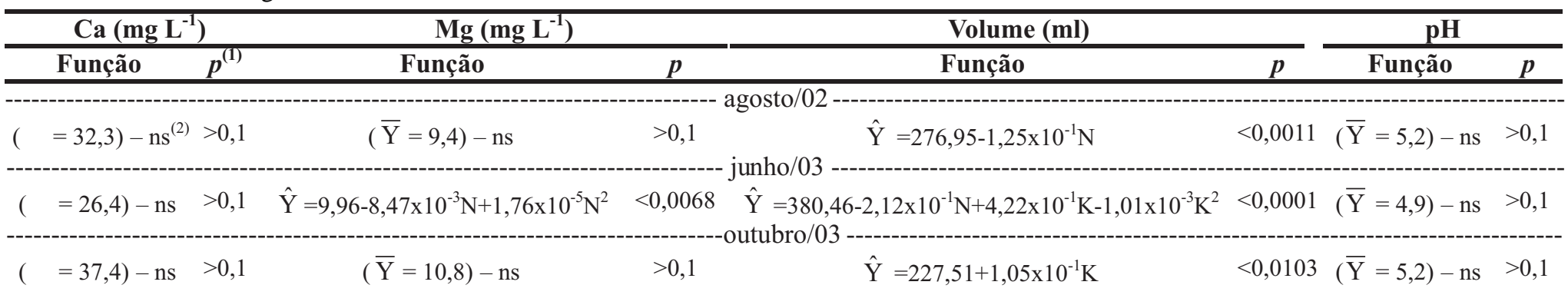

(1) Probabilidade de erro tipo I associada ao teste $\mathrm{F}$ (significância do modelo); ${ }^{(2)}$ valor entre parêntesis corresponde à média da variável; ajuste não significativo ( $\left.p>0,1\right)$.

TABELA 4 - Efeito da aplicação de N, Pe K (doses em kg ha-1 de N, $\mathrm{P}_{2} \mathrm{O}_{5}$ e $\mathrm{K}_{2} \mathrm{O}$ ) no conteúdo de sólidos solúveis totais, acidez titulável e ratio da água de coco em três épocas de amostragem.

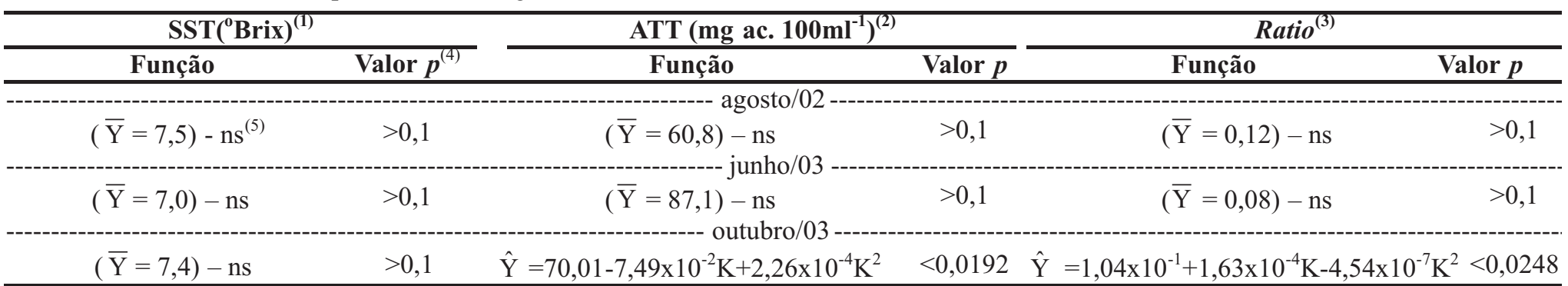

(1) Sólidos solúveis totais ( $\left.{ }^{\circ} \mathrm{Brix}\right) ;{ }^{(2)}$ acidez total titulável (mg de ácido málico/100 $\mathrm{ml}$ de água); ${ }^{(3)}$ ratio=SST/ATT; ${ }^{(4)}$ probabilidade de erro tipo I associada ao teste $\mathrm{F}$ (significância do modelo); ${ }^{(5)}$ valor entre parêntesis corresponde à média da variável; ajuste não significativo $(p>0,1)$.

TABELA 5 - Efeito da aplicação de $\mathrm{N}, \mathrm{Pe} \mathrm{K}$ (doses em kg ha-1 de N, $\mathrm{P}_{2} \mathrm{O}_{5}$ e $\mathrm{K}_{2} \mathrm{O}$ ) no número de frutos por cacho, massa média dos frutos em três épocas e produção mensal em duas épocas de amostragem.

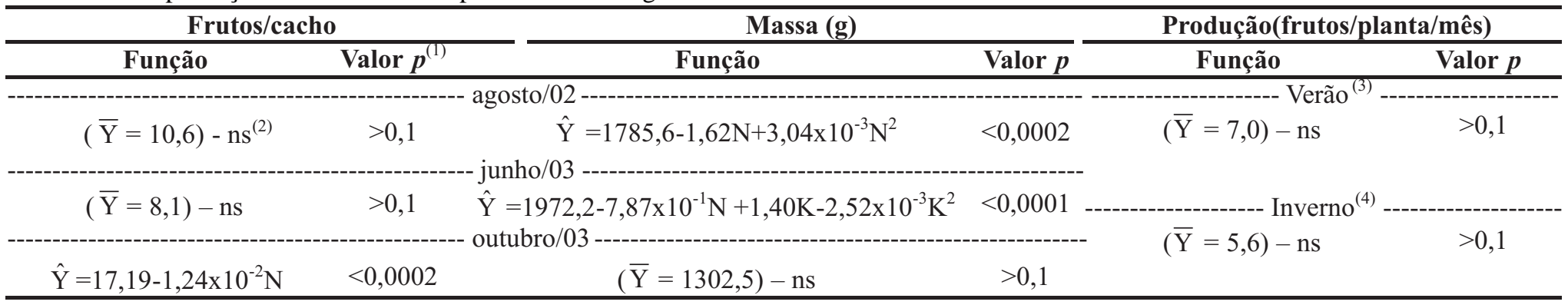

\footnotetext{
(1) Probabilidade de erro tipo I associada ao teste $\mathrm{F}$ (significância do modelo);

${ }^{(2)}$ valor entre parêntesis corresponde à média da variável; ajuste não significativo $(p>0,1)$;

${ }^{(3)}$ cachos emitidos entre maio e setembro, enchimento de frutos na primavera/verão;

(4) cachos emitidos entre janeiro e março, enchimento de frutos no outono/inverno.
}

valores ainda abaixo do teto de produção de coqueiro-anão. Diferentemente, Sobral (2004) observou efeitos positivos da aplicação de $\mathrm{N}$ e K por fertirrigação na quantidade de frutos produzidos por coqueiro 'anão-verde' com oito anos de idade, colhendo até 150 frutos/planta/ ano. Outro fator que poderia ter determinado esta pequena resposta à adubação é o tempo que normalmente as plantas levam para refletir os efeitos da aplicação de fertilizantes na produção. Segundo resultados de pesquisas de médio e longo prazos compilados por Sobral (1998), as respostas à aplicação de fertilizantes só costumam aparecer depois de dois anos. No caso deste trabalho, os tratamentos com as diferentes doses de NPK começaram a ser aplicados no verão de 2000-2001.

Apesar de o teor de P disponível no solo ser baixo (Tabela 1), a adubação fosfatada teve pouco efeito na qualidade e na quantidade de frutos produzidos (Tabelas 2 a 5). Khan et al. (1985), após extensa revisão sobre aplicação de $\mathrm{P}$ em coqueiro, concluíram que este nutriente, apesar de sua importância fisiológica, raramente limita a produção, classificando esta cultura como pouco responsiva à adubação fosfatada.

A produção mensal de frutos apresentou significativa sazonalidade (Tabela 5). Cachos emitidos entre maio e setembro e com enchimento de frutos na primavera/verão, produziram cerca de $25 \%$ mais frutos do que aqueles emitidos entre janeiro e março e que se desenvolveram no outono/inverno. Passos (1999) destacou que o coqueiro é uma "planta eminentemente tropical" e alertou que as baixas temperaturas e umidade do ar que ocorrem na região Sudeste, na época de seca, seriam fatores limitantes à cultura.

\section{CONCLUSÕES}

1. Os teores de $\mathrm{P}$ e $\mathrm{K}$ na água de coco aumentaram em resposta à aplicação de $\mathrm{P}$ e K. Entretanto, a aceitabilidade não foi influenciada pelos tratamentos de adubação.

2. A produção respondeu negativamente à aplicação de $\mathrm{N}$, ocorrendo diminuição no volume de água e massa média dos frutos e quantidade de frutos por cacho. De outra forma, estas características responderam positivamente à aplicação de potássio.

3. Atributos físico-químicos da água de coco não foram influenciados pela aplicação de $\mathrm{N}$ ou $\mathrm{P}$.

4. Excetuando-se a variação no teor de $\mathrm{P}$ da água, os efeitos da adubação fosfatada na produção de frutos não foram significativos.

\section{REFERÊNCIAS}

INSTITUTO ADOLFO LUTZ. Normas analíticas do InstitutoAdolfo Lutz. 3.ed. São Paulo: Instituto Adolfo Lutz, 1985. v.1, 533p. 
KHAN, H.H.; BIDDAPPA, C.C.; JOSHI, O.P. A review of Indian work on phosphorus nutrition of coconut. Journal of Plantation Crops, Kasarogod, v.13, n.1, p.11-21, 1985.

LIMA, E.; SILVA, J.Q.; MORAIS, R.F.; BARBOSA, M.A.D.; D’AGOSTINI, F.S. Caracterização físico-química da água de fruto do coqueiro anão verde, produzido no litoral sul do Rio de Janeiro. In: FERTBIO 2004, Lages. Anais... Lages: UDESC/SBCS, 2004. CDRom.

MAGAT, S.S. Coconut. In: World fertilizer use manual. International Fertilizer Association (IFA). 2005. Disponível em: <http:// www.fertilizer.org/ifa/publicat/html/pubman/coconut.htm $>$ Consultado em: 11 abr. 2005.

MALAVOLTA, E.; VITTI, G.C.; OLIVEIRA, S.A. Avaliação do estado nutricional das plantas: princípios e aplicações. 2. ed. Piracicaba: POTAFOS. 1997.319p.

PASSOS, E.E.M. Ecofisiologia do coqueiro. In: SÃO JOSÉ, A.R.; SOUZA, I.V.B.; MOURA, J.I.L.; REBOUÇAS, T.N.H. (Ed.). Coco: produção e mercado. Vitória da Conquista: DFZ/UESB, 1999. p.36-43.

RAIJ, B. van; ANDRADE, J.C.; CANTARELLA, H.; QUAGGIO, J.A. (Ed.) Análise química para avaliação da fertilidade de solos tropicais. Campinas: IAC, 2001.285p.
SILVA, F. C. (Ed.). Manual de análises químicas de solos, plantas e fertilizantes. Brasília: EMBRAPA, 1999.370p.

SOBRAL, L.F. Fertirrigação do coqueiro anão verde com N e K no platô de Neópolis. In: FERTBIO 2004, Lages. Anais... Lages: UDESC/SBCS, 2004. CD-ROM.

SOBRAL, L.F. Nutrição e adubação do coqueiro. In: FERREIRA, J.M.S.; WARWICK, D.R.N.; SIQUEIRA, L.A. (Ed.) A cultura do coqueiro no Brasil. 2.ed. Aracaju: EMBRAPA-CPATC, 1998. p.129-157.

SOBRAL, L.F. Nutrição e adubação. In: FONTES, H.R.; RIBEIRO, F.E.; FERNANDES, M.F. (Ed.). Coco: produção. Brasília: EMBRAPA, 2003. p.44-52.

SOBRAL, L.F.; LEAL, M.L.S. Resposta do coqueiro à adubação com uréia, superfosfato simples e cloreto de potássio em dois solos no nordeste do Brasil. Revista Brasileira de Ciência do Solo, Viçosa, v.23, n.1, 1999.

TEIXEIRA, L.A.J.; BATAGLIA, O.; BUZETTI, S.; FURLANI JR., E. Adubação com NPK em coqueiro anão-verde (Cocos nucifera L.) atributos químicos do solo e nutrição das plantas. Revista Brasileira de Fruticultura, Jaboticabal, v.1, n.27, p.115-119, 2005. 